NASA Technical Memorandum 102142

ICOMP-89-16

\title{
Calculation of Turbulence-Driven Secondary Motion in Ducts With Arbitrary Cross Section
}

\author{
A.O. Demuren \\ Institute for Computational Mechanics in Propulsion \\ Lewis Research Center \\ Cleveland, Ohio
}

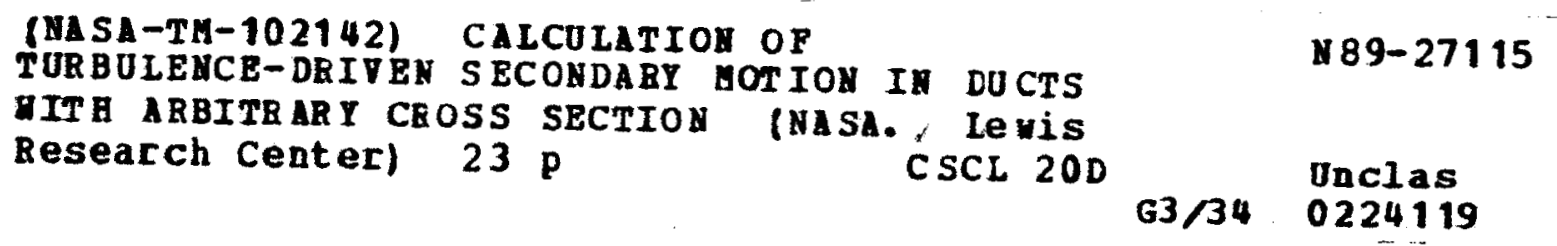

July 1989

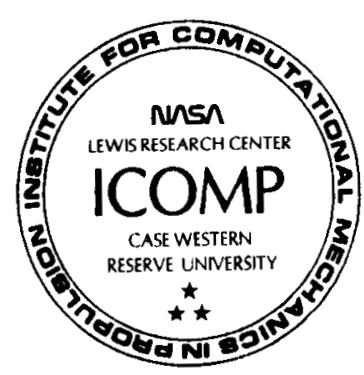




\title{
CALCULATION OF TURBULENCE-DRIVEN SECONDARY MOTION IN DUCTS WITH ARBITRARY CROSS-SECTION
}

\author{
A.O. Demuren* \\ Institute for Computational Mechanics in Propulsion \\ Lewis Research Center \\ Cleveland, Ohio 44135
}

\begin{abstract}
Calculation methods for turbulent duct flows are generalised for ducts with arbitrary crosssections. The irregular physical geometry is transformed into a regular one in computational space, and the flow equations are solved with a finite-volume numerical procedure. The turbulent stresses are calculated with an algebraic stress model derived by simplifying model transport equations for the individual Reynolds stresses. Two variants of such a model are considered in the present study. These procedures enable the prediction of both the turbulence-driven secondary flow and the anisotropy of the Reynolds stresses, in contrast to some of the earlier calculation methods. Model predictions are compared to experimental data for developed flow in triangular duct, trapezoidal duct and a rod-bundle geometry. The correct trends are predicted, and the quantitative agreement is mostly fair. The simpler variant of the algebraic stress model procured better agreement with the measured data.
\end{abstract}

*On leave from University of Lagos, Lagos, Nigeria. Work funded under Space Act Agreement C99066G. 
NOMENCLATURE

\begin{tabular}{|c|c|}
\hline$\overline{\mathrm{B}}$ & half-width of duct \\
\hline$c_{1}$ & constant in algebraic stress model \\
\hline$c_{\mu}, c_{\epsilon 1}, c_{\epsilon 2}$ & $k-\epsilon$ turbulence model constants \\
\hline $\mathrm{D}$ & determinant of the Jacobian of the transformation \\
\hline f & near-wall proximity function in algebraic stress model \\
\hline G & rate of production of turbulent kinetic energy \\
\hline $\mathbf{H}$ & half-height of duct \\
\hline $\mathbf{J}$ & Jacobian of the transformation $y^{j}=y^{j}\left(x^{j}\right)$ \\
\hline $\mathbf{k}$ & turbulent kinetic energy \\
\hline $\mathbf{L}$ & mixing length \\
\hline $\mathbf{P}$ & pressure \\
\hline$q_{\phi}$ & stress or flux of variable $\Phi$ \\
\hline s & distance from point in flow to a point on wall \\
\hline$S_{\phi}$ & source term for dependent variable $\Phi$ \\
\hline$U_{b}$ & bulk velocity \\
\hline$U_{o}$ & maximum velocity \\
\hline$U_{*}$ & friction velocity \\
\hline$U_{i}$ or $U, V, W$ & cartesian velocity components; streamwise, lateral, vertical \\
\hline$\overline{u_{1}^{2}}, \overline{u_{2}^{2}}, \overline{u_{3}^{2}}$ & Reynolds normal stresses in cartesian directions \\
\hline$\overline{u_{1} u_{2}}, \overline{u_{1} u_{3}}, \overline{u_{2} u_{3}}$ & Reynolds shear stresses ( 2 primary and 1 secondary) \\
\hline$x^{j}$ & curvilinear coordinates \\
\hline$y^{j}$ & cartesian coordinates \\
\hline$<y^{2}>$ & square of the average distance from a point to all walls \\
\hline$\alpha, \beta, \gamma$ & constants in algebraic stress model \\
\hline$\alpha_{j}^{i}$ & metric coefficients of transformation \\
\hline$\delta_{i j}$ & Kronecker delta \\
\hline$\epsilon$ & rate of dissipation of turbulent kinetic energy \\
\hline$\theta$ & angle \\
\hline$\kappa$ & von Karman constant \\
\hline$\mu_{t}$ & turbulent eddy viscosity \\
\hline$\rho$ & density \\
\hline$\sigma_{\phi}$ & turbulent Prandtl/Schmidt number for $\Phi$ \\
\hline$\tau$ & wall shear stress \\
\hline$\Phi$ & General representation of dependent variable \\
\hline \multicolumn{2}{|l|}{ Superscripts } \\
\hline 1 & longitudinal direction \\
\hline 2,3 & vertical and lateral directions \\
\hline
\end{tabular}




\section{INTRODUCTION}

Secondary motion of Prandtl's first kind is encountered in curved passages, where centrifugal forces act at an angle to the streamwise direction. It is pressure-driven, with magnitudes ranging from $10-40 \%$ of the bulk streamwise velocity. It usually leads to a first-order perturbation of the main flow properties, and there is no real difficulty in calculating it. On the other hand, secondary motion of Prandtl's second kind is turbulence-driven and is believed to result from the anisotropy of the turbulent stresses. It is associated with internal or external turbulent flows in the presence of streamwise corners. Its magnitude has been observed to be mostly between 1-4 \% of the bulk mean velocity in most straight ducts with non-circular cross-section, but the effects on wall shear stress distribution and heat transfer rates are quite significant. Some form of Reynolds stress modelling is usually required in order to calculate this type of secondary motion.

Probably the first qualitative data on turbulence-driven secondary motion in non-circular ducts were presented by Nikuradse[1], using flow visualisation techniques. Actual quantitative measurements were presented by Hoagland[2] aboout 30 years later. Several detailed measurements have subsequently been presented. A comprehensive review of experimental data for ducts with regular cross-section can be found in Demuren and Rodi [3]. Additional data for irregularly-shaped channels are reported by Rodet [4], Aly et al [5], and Seale [6], for trapezoidal, triangular and simlated rod-bundle-type cross-sections, respectively.

The first calculation of secondary flow in straight non-circular ducts was due to Launder and Ying [7] (hereafter denoted LY). Their method is based on approximating the Reynolds stresses with algebraic expressions by simplifying the corresponding transport equations proposed by Hanjalic and Launder [8] This model has subsequently been employed, sometimes with modifications in numerous studies [9-12] to calculate secondary flows in ducts with regular as well as gemetrical crosssections. In all these studies, it has been possible to predict the magnitudes of the secondary motion fairly well, but always at the expense of the Reynolds stress distributions. The anisotropy of the secondary normal stresses are usually underpredicted by an order of magnitude. This discrepancy has been discussed by various authors, including Demuren and Rodi [3], who showed that certain terms which are neglected in LY model could in fact be larger in magnitude than terms which are included. In order to avoid this shortcoming, Seale [6] in the calculation of developed flow in a rod-bundle-type configuration, utilises an algebraic model for the streamwise vorticity source, based on the correlation of measured turbulence data. Very good agreement between measured and computed velocity and wall shear stress distributions are reported. The prospects of such a method as a general simulation technique for arbitrary geometries is uncertain. The reported calculations are perfomed on a fairly coarse cartesian grid, using a method which approximates the curved wall with steps. The influence of such a boundary treatment on the total flow is uncertain, but it doubtful that it can produce a true picture of the flow near the curved wall. A truly general computational method should employ a boundary-fitted coordinate system.

Naot and Rodi [13] (hereafter denoted NR) calculated flows in rectangular ducts using an algebraic stress model developed from the Reynolds stress model of Launder Reece and Rodi [14]. The method was subsequently extended by Demuren and Rodi [3] (hereafter denoted DR). To put the various models in perspective. LY model is derived from the Reynolds stress model of Hanjalic and Launder [8]. The latter is a precursor to the more complete Reynolds stress model of Launder, Reece and Rodi [14]. The LY model neglects all terms involving secondary velocity gradients. NR model is based on the more complete Reynolds stress model and approximates terms 
involving secondary velocity gradients by eddy viscosity type relations. DR model is also based on the same Reynolds stress model, but uses the full derivation. In all the models the algebraic stress relations are derived from the partial-differential equations governing the Reynolds stress transport by neglecting the convection and diffusion terms or relating them to those of the turbulent kinetic energy. This approximation is adequate in straight wall bounded flows, but would be inadequate for free shear flows or flows with high mean shear rates in which the convection and diffusion terms assume more significant roles. As may be expected, DR model is more difficult to implement than the other two. It is also much more sensitive to the solution procedure, because of the much stronger coupling between the Reynolds stresses. Both NR and DR model produce correct simulation of mean flow and Reynolds stresses in developing and fully developed flow in square and rectangular ducts $[3,15]$. In fully developed flow situations the secondary flow level predicted with the former agree better with experimental data.

In the present study, NR and DR models are generalised for any curvilinear coordinate system and then applied to predict developed turbulent flow in straight ducts with arbitrary cross-sections. The governing equations are transformed into a general curvilinear coordinate system, but the cartesian velocity components are retained as dependent variables, following the practice of Rhie and Chow [16].

\section{MATHEMATICAL MODEL}

The time-averaged, three-dimensional, steady-state equations governing turbulent flows may be expressed in curvilinear tensor, for a general property $\Phi$ as :

$$
\frac{\partial}{\partial x^{j}}\left[D\left(\rho U_{m} \Phi-q_{\phi m}\right) \alpha_{m}^{j}\right]=D S_{\phi}
$$

where $x^{j}$ is a general curvilinear coordinate system related to the cartesian coordinate system $y^{j}$ by the transformation $y^{j}=y^{j}\left(x^{j}\right)$.

$\mathrm{D}$ is the determinant of the Jacobian of the transformation; i.e., $D=|J|$, with

$$
J=\left(\begin{array}{lll}
\frac{\partial y^{1}}{\partial x^{1}} & \frac{\partial y^{1}}{\partial x^{2}} & \frac{\partial y^{1}}{\partial x^{3}} \\
\frac{\partial y^{2}}{\partial x^{1}} & \frac{\partial y^{2}}{\partial x^{2}} & \frac{\partial y^{2}}{\partial x^{3}} \\
\frac{\partial y^{3}}{\partial x^{1}} & \frac{\partial y^{3}}{\partial x^{2}} & \frac{\partial y^{3}}{\partial x^{3}}
\end{array}\right)
$$

$\alpha_{j}^{i}$, also called the metrics, are the elements of the Jacobian $J^{-1}$ of the inverse transformation, $x^{j}=x^{j}\left(y^{j}\right)$, i.e., $\alpha_{j}^{i}=\frac{\partial x^{i}}{\partial y^{j}}$.

$U_{m}$ represents the cartesian velocity components.

$q_{\phi m}$ and $S_{\phi}$ are respectively fluxes/stresses and source terms for the corresponding $\Phi$. They may be expressed as : 


\begin{tabular}{|c|c|c|c|}
\hline Conservation Equation & $\Phi$ & $q_{\phi}$ & $S_{\phi}$ \\
\hline Continuity & 1 & 0 & 0 \\
\hline Momentum Components & $U_{i}$ & $-P \delta_{m i}-\rho \overline{u_{m} u_{i}}+\mu\left(\frac{\partial U_{i}}{\partial x^{n}} \alpha_{m}^{n}+\frac{\partial U_{m}}{\partial x^{l}} \alpha_{i}^{l}\right)$ & 0 \\
\hline Turbulent kinetic energy & $k$ & $\frac{\mu_{t}}{\sigma_{k}} \frac{\partial k}{\partial x^{n}} \alpha_{m}^{n}$ & $G-\rho \epsilon$ \\
\hline Dissipation rate of $\mathbf{k}$ & $\epsilon$ & $\frac{\mu_{t}}{\sigma_{\epsilon}} \frac{\partial \epsilon}{\partial x^{n}} \alpha_{m}^{n}$ & $\frac{\epsilon}{k}\left(c_{\epsilon 1} G-c_{\epsilon 2} \rho \epsilon\right)$ \\
\hline
\end{tabular}

where, $\delta_{m i}$ is the Kronecker delta

and, $G$ is the rate of production of turbulent kinetic energy by the interaction of turbulent stresses with mean shear, and is given by :

$$
G=\mu_{t}\left(\frac{\partial U_{i}}{\partial x^{n}} \alpha_{j}^{n}+\frac{\partial U_{j}}{\partial x^{m}} \alpha_{i}^{\mathrm{n}}\right) \frac{\partial U_{i}}{\partial x^{n}} \alpha_{j}^{n}
$$

The turbulent eddy viscosity $\mu_{t}$ is given by the standard $k-\epsilon$ model as

$$
\mu_{t}=c_{\mu} \rho \frac{k^{2}}{\epsilon}
$$

The empirical constants are:

$$
c_{\epsilon 1}=1.44, c_{\epsilon 2}=1.92, \sigma_{k}=\sigma_{\epsilon}=1.225
$$

In the above, we have used the Einstein summation convention for repeated indices to write in compact form. Thus, for the 3-dimensional flow under consideration,

$$
a_{m}^{i} b_{j}^{m}=a_{1}^{i} b_{j}^{1}+a_{2}^{i} b_{j}^{2}+a_{3}^{i} b_{j}^{3}
$$

The equations can be simplified if we consider only straight channels with constant cross-section in the streamwise direction $\left(y^{1}=x^{1}\right)$. Then, we can use parabolic assumptions to decouple the pressure gradient in the streamwise direction from those in the cross-plane, and also neglect the derivatives with respect to $x^{1}$ or $y^{1}$, in all but the convection terms. They will of course be zero throughout in fully developed flow, and the equations become 2-dimensional.

The major difficulty in solving the set of equations is to determine the distribution of the Reynolds stresses $-\rho \overline{u_{i} u_{j}}$. Following Demuren and Rodi $[3,15]$, we can write the algebraic equation for the Reynolds stresses as :

$$
\begin{gathered}
-(1-\alpha)\left[\overline{u_{i} u_{l}} \frac{\partial U_{j}}{\partial x^{m}} \alpha_{l}^{m}+\overline{u_{j} u_{l}} \frac{\partial U_{i}}{\partial x^{m}} \alpha_{l}^{m}\right] \\
+\beta\left[\overline{u_{i} u_{l}} \frac{\partial U_{l}}{\partial x^{m}} \alpha_{j}^{m}+\overline{u_{j} u_{l}} \frac{\partial U_{l}}{\partial x^{m}} \alpha_{i}^{m}\right] \\
-\gamma k\left[\frac{\partial U_{i}}{\partial x^{m}} \alpha_{j}^{m}+\frac{\partial U_{j}}{\partial x^{m}} \alpha_{i}^{m}\right]-\frac{\epsilon}{k}\left[c_{1} \overline{u_{i} u_{j}}+\frac{2}{3}\left(1-\alpha-\beta-c_{1}\right) \delta_{i j} k\right]=0
\end{gathered}
$$

Equation (6) shows very strong coupling between the Reynolds stresses, and in order to stabilise the solution process only the secondary stresses $\overline{u_{2}^{2}}, \overline{u_{3}^{2}}$ and $\overline{u_{2} u_{3}}$ are obtained from it. The primary normal and shear stresses are calculated from the turbulent kinetic energy and standard eddy viscosity relations. The resulting set of algebraic stress relations are:- 


$$
\begin{gathered}
\overline{u_{1}^{2}}=2 k-\overline{u_{2}^{2}}-\overline{u_{3}^{2}} \\
-\overline{u_{1} u_{2}}=\frac{\mu_{t}}{\rho} \frac{\partial U_{1}}{\partial x^{m}} \alpha_{2}^{m} \\
-\overline{u_{1} u_{3}}=\frac{\mu_{t}}{\rho} \frac{\partial U_{1}}{\partial x^{m}} \alpha_{3}^{m} \\
\overline{u_{2}^{2}}=\frac{2 k}{c_{1} \epsilon}\left[\left(\alpha+\beta+c_{1}-1\right)+\beta \overline{u_{1} u_{2}} \frac{\partial U_{1}}{\partial x^{m}} \alpha_{2}^{m}\right. \\
-\overline{u_{2} u_{3}}\left\{(1-\alpha) \frac{\partial U_{2}}{\partial x^{m}} \alpha_{3}^{m}-\beta \frac{\partial U_{3}}{\partial x^{m}} \alpha_{2}^{m}\right\} \\
\left.-\gamma k \frac{\partial U_{2}}{\partial x^{m}} \alpha_{2}^{m}\right] /\left[1+\frac{2 k}{c_{1} \epsilon}(1-\alpha-\beta) \frac{\partial U_{2}}{\partial x^{m}} \alpha_{2}^{m}\right] \\
\overline{u_{3}^{2}}=\frac{2 k}{c_{1} \epsilon}\left[\left(\alpha+\beta+c_{1}-1\right)+\beta \overline{u_{1} u_{3}} \frac{\partial U_{1}}{\partial x^{m}} \alpha_{3}^{m}\right. \\
-\overline{u_{2} u_{3}}\left\{(1-\alpha) \frac{\partial U_{3}}{\partial x^{m}} \alpha_{2}^{m}-\beta \frac{\partial U_{2}}{\partial x^{m}} \alpha_{3}^{m}\right\} \\
\left.-\gamma k \frac{\partial U_{3}}{\partial x^{m}} \alpha_{3}^{m}\right] /\left[1+\frac{2 k}{c_{1} \epsilon}(1-\alpha-\beta) \frac{\partial U_{3}}{\partial x^{m}} \alpha_{3}^{m}\right] \\
\overline{u_{2} u_{3}}=\frac{k}{c_{1} \epsilon}\left[\beta\left(\overline{u_{1} u_{3}} \frac{\partial U_{1}}{\partial x^{m}} \alpha_{2}^{m}+\overline{u_{1} u_{2}} \frac{\partial U_{1}}{\partial x^{m}} \alpha_{3}^{m}\right)\right. \\
\left.\left.-\overline{u_{3}^{2}}+\gamma k\right\} \frac{\partial U_{3}}{\partial x^{m}} \alpha_{2}^{m}-\left\{(1-\alpha) \overline{u_{3}^{2}}-\beta \overline{u_{2}^{2}}+\gamma k\right\} \frac{\partial U_{2}}{\partial x^{m}} \alpha_{3}^{m}\right]
\end{gathered}
$$

In equations $(10-12)$ wall proximity effects are accounted for by making the empirical coefficients functions of a dimensional distance from the walls, thus :

$$
\alpha=0.7636-0.06 f ; \beta=0.1091+0.06 f ; \gamma=0.182 ; c_{1}=1.5-0.50 f
$$

where $f$ is the wall-proximity function given by :

$$
f=\frac{L^{2}}{\left\langle y^{2}\right\rangle} ; \quad\left\langle y^{2}\right\rangle=\frac{2}{\pi} \int_{0}^{2 \pi} \frac{d \theta}{s^{2}}
$$

$L=\frac{c_{\mu}^{3 / 4}}{\kappa} \frac{k^{3 / 2}}{\epsilon}$ is the length scale of the turbulent motion at the grid node, and $<y^{2}>1 / 2$ is the average distance from all walls.

In the NR model, equations $(10-12)$ are further simplified by setting the denominator to unity and approximating terms involving secondary velocity gradients by eddy-viscosity-type relations as:

$$
\begin{gathered}
\frac{k}{c_{1} \epsilon}\left[\overline{u_{2} u_{3}}\left\{(1-\alpha) \frac{\partial U_{2}}{\partial x^{m}} \alpha_{3}^{m}-\beta \frac{\partial U_{3}}{\partial x^{m}} \alpha_{2}^{m}\right\}-\gamma k \frac{\partial U_{2}}{\partial x^{m}} \alpha_{2}^{m}\right] \simeq \frac{\mu_{t}}{\rho} \frac{\partial U_{2}}{\partial x^{m}} \alpha_{2}^{m} \\
\frac{k}{c_{1} \epsilon}\left[\overline{u_{2} u_{3}}\left\{(1-\alpha) \frac{\partial U_{3}}{\partial x^{m}} \alpha_{2}^{m}-\beta \frac{\partial U_{2}}{\partial x^{m}} \alpha_{3}^{m}\right\}-\gamma k \frac{\partial U_{3}}{\partial x^{m}} \alpha_{3}^{m}\right] \simeq \frac{\mu_{t}}{\rho} \frac{\partial U_{3}}{\partial x^{m}} \alpha_{3}^{m} \\
\frac{k}{c_{1} \epsilon}\left[\left\{(1-\alpha) \overline{u_{2}^{2}}-\beta \overline{u_{3}^{2}}+\gamma k\right\} \frac{\partial U_{3}}{\partial x^{m}} \alpha_{2}^{m}\right.
\end{gathered}
$$




$$
\left.-\left\{(1-\alpha) \overline{u_{3}^{2}}-\beta \overline{u_{2}^{2}}+\gamma k\right\} \frac{\partial U_{2}}{\partial x^{m}} \alpha_{3}^{m}\right] \simeq \frac{\mu_{t}}{\rho}\left(\frac{\partial U_{3}}{\partial x^{m}} \alpha_{2}^{m}+\frac{\partial U_{2}}{\partial x^{m}} \alpha_{3}^{m}\right)
$$

This practice has a stabilising effect on the solution procedure, since they can now be treated implicitly as diffusion terms in the momentum equations for the secondary velocity components. An analysis of the implications of these approximations, performed by Demuren and Rodi [3] for a square duct flow, has shown that the major effect is on the secondary shear stress calculation, of which equation (17) is a dominant part. That term is essentially reduced by a factor of $2-3$. However, the secondary shear stress acts a sink of the streamwise vorticity, so that a reduction in its magnitude would lead to an increase in the secondary motion. This explains why the NR model usually produces stronger secondary motion than the DR model [3]. The terms in equations (15) and (16) are small compared to the rest of the terms in equations (10) and (11), so that the effects of approximating them is minimal. If the calculations are performed with the secondary velocity terms in equations (15) - (17) neglected completely, as is done in LY model and derivatives thereof, the secondary motion will grow indefinitely and no stable solution can be obtained. In this case, a stable solution can only be obtained through an ad-hoc reduction in the anisotropy of the secondary normal stresses, which is the source of streamwise vorticity.

\section{SOLUTION PROCEDURE AND BOUNDARY CONDITIONS}

The differential equations to be solved are parabolic in the streamwise direction so that influences only travel from upstream to downstream. An efficient once-through forward marching procedure can thus be used, starting from given inlet conditions. The procedure is essentially an incorporation of a non-staggered grid arrangement into the SIMPLE algorithm of Patankar and Spalding [17]. Where the grid is not orthogonal, cross-derivative terms lead to the formation of a 9-point computational molecule for the 2-dimensional plane. In the present study, these terms are treated explicitly in the equations for the velocity components. This practice proved not to be so robust in the solution of the pressure correction equation, which is the most critical for stability and convergence. We therefore employ a 9-point pressure-correction equation which is an extension of Stone's strongly implicit scheme as proposed by Peric [18]. A comparative study [19] of various solution schemes, including coupled and decoupled methods, for two-dimensional flow problems on a non-orthogonal grid shows the present arrangement to be the most effective.

Four types of boundaries are usually encountered, at which conditions must be specified for the dependent variables. At inlet planes, known (or simulated) conditions are specified for all variables; usually uniform values for the primary velocity and the turbulent quantities, and zero for the secondary velocity components. No specific conditions are required at outflow boundaries, since the equations are parabolic. Along a symmetry plane, the velocity component normal to the surface is set to zero. Further, the gradient of all other variables is zero in the direction normal to the surface. At solid walls, the velocity component normal to the surface is also set to zero, but rather than carry out the solution all the way down to the wall, we employ the wall-function method [3] to specify the values of the velocity components parallel to the surface, and the turbulence quantities, along the first line of grid nodes away from the wall. These grid nodes are usually arranged to be in the fully turbulent region, where the logarithmic law of the wall may be assumed to be applicable. 


\section{RESULTS AND DISCUSSION}

\subsection{Test Cases}

Three test cases are considered in the present study. These coincide with flow in ducts with cross-sections requiring non-rectangular grids, for which experimental data could be found in the literature. In each case, the channel cross-section is constant in the streamwise direction, and the flow is nominally fully-developed.

Case $I$ is the flow in an equilateral triangular duct (fig. 1) studied experimentally by Aly et al [5] at Reynolds number of 53,000, based on the bulk velocity and the hydraulic diameter. Measurements were made in one-sixth of the duct cross-section at a plane 133 diameters from the inlet. Case II is the flow, at a Reynolds number of 240,000 , in a trapezoidal channel with two sides parallel and two slanting sides at angles $75^{\circ}$ and $105^{\circ}$ (fig. 2). Rodet [4] presents measurements of the velocity distribution and the Reynolds stresses at a cross-stream plane 50 diameters from the inlet. Judging by the review of duct flow data, this would barely be long enough for full flow development. The cross-section of the channel for case III is shown in fig. 3. It was studied experimentally by Seale [6] to simulate approximately flows encountered in rod-bundle geometries. Measurements were made in a symmetric quadrant at a Reynolds number of 200,000 and 120 diameters.

\subsection{Computational Details}

In the calculations, advantage is taken of the inherent symmetry of the flow to reduce the size of the computational domain. Thus, we compute only a third of the triangular duct half of the trapezxoidal duct, and a quarter of the rod-bundle-type geometry. In each case, the grid is generated algebraically using transfinite mapping of the boundary points. Where necessary, a third-order smoother based on Newton's divided difference formula is applied. Although orthogonality is desirable, it is not a requirement of the present solution procedure. The sole constraint applied is that the normal distance of the first set of grid nodes, nearest to the wall should lie in the range $y^{+}=30-200$.

Since all the present test cases apply to fully-developed flow, the grid distribution in the streamwise direction is unimportant. The practice adopted here is to take an infinitely large step size, and then use a relaxation method to obtain a converged solution. An underrelaxation method with a maximum value of 0.5 is applied to all variables. A typical computation on a $40 \times 40$ grid required about 600 iterations to converge to a normalised residual norm of $10^{-6}$ when the NR model is used. DR model calculations require 200-300 iterations more. Grid dependency tests are performed by doubling the total number of grid nodes, i.e., an increase by a factor of $\sqrt{2}$ in each direction, and comparing the results. These tests show that a $26 \times 26$ grid is sufficient for case I. Case II requires a $18 \times 36$ grid, and case III a $40 \times 40$ grid.

\subsection{Comparison with Experimental Data}

\subsubsection{Triangular Duct}

The measured wall shear stress distribution is compared to present predictions with the $k-\epsilon, \mathrm{NR}$ and DR models in fig. 4. Both the NR and DR models capture the trend of the data towards more uniformity, though the former has the better agreement. The $k-\epsilon$ model predicts much wider 
variation in wall shear stress. These can be explained with the results of the predicted secondary flow presented in figs. 5 and 6 . This flow moves towards the corners along the corner bisector and returns along the walls towards the wall bisectors. Whilst the NR and DR models predict the correct trend, the $k-\epsilon$ model produces no secondary motion. This motion leads to a redistribution of the wall shear stress by moving high velocity fluid from the central part of the duct to the corners thereby increasing the shear. In contrast, the shear stress near the wall bisector is reduced by the slower moving fluid convected along the wall. The influence of this secondary motion on the streamwise velocity distribution is shown in fig. 7. The experiments show strong bulging of the velocity contours towards the corners. The predictions mirror this effect to varying degrees. The $k-\epsilon$ model predicts nearly circular contours (not shown). Fig. 8 shows similar comparisons for the turbulent kinetic energy. The normal stresses are compared in fig. 9. Both the NR and DR models predict the correct degree of anisotropy.

\subsubsection{Trapezoidal Duct}

The present predictions with both NR and DR models show that there are two eddies in each corner. The one stradling the longer side is much larger, as may be expected. Fig. 10 compares the predicted contours of the streamwise velocity component to the measurements of Rodet [4]. NR model predicts the bulging of the contours towards the corners to the correct degree, though there is some deviation between the actual levels along the centreline. DR model predictions agree better with the magnitudes along the centreline, but produces too little bulging of the contours towards the corners, due to its characteristic of producing weaker secondary motion. Predictions of the turbulent kinetic energy, $k$, are compared in fig. 11. The effect of the secondary motion in distorting the distribution of $\mathrm{k}$ is much stronger. Correspondingly, the differences between the predictions are magnified. Again NR model shows the correct trends overall, but DR model has better agreement with centreline values.

The source of streamwise vorticity is the anisotropy of the secondary normal turbulent stresses. A measure of this anisotropy is shown in fig. 12. The present prediction (NR model) compares quite well with the data. The predictions by Nakayama et al [12], which is characteristic of those based on the LY model show magnitudes which are at least an order of magnitude too low. This observation has, of course, been widely discussed in the literature without isolating the true cause.

\subsubsection{Rod-Bundle Geometry}

Case III represents the most complicated geometry of all the test cases computed in this study. There are 5 corners, all different from each other. We have combinations of intersections of straight walls, curved walls and symmetry planes. The geometry certainly poses a severe test on the predictive ability of the present mathematical procedure and turbulence model. There are, however significant differences from the usual rod-bundle geometries, which contain no wall/wall intersections. Figure 13 compares the wall shear stress distributions predicted with the three turbulence models to measured data of Seale [6]. The data shows nearly uniform wall shear stresses, away from the corners. This effect is, of course, due to the equalizing action of the the secondary motion. The $k-\epsilon$ model cannot simulate this effect since it does not produce any secondary motion. DR model does produce a more uniform distribution, but the secondary motion still appears to be too weak. NR model predicts the correct level of shear stress, but this has a wavy distribution, not apparent in the measurements. It is not certain if the waviness is physical, but the results 
have been reproduced with the $\mathrm{NR}$ model, using a different numerical procedure, so it does not appear to be numerical in origin. The measurements do not appear to be sufficiently detailed to provide definitive answer. Examination of the lateral velocity data, presented in fig. 14, shows very strong periodic variations along the verticals. The predictions do simulate this behaviour. As usual, the NR model performs better. The peaks in the predicted wall shear stress profiles are seen to coincide with the maxima in the velocity component directed towards the vertical wall. Thus the data suggests the action of the secondary motion near the vertical wall could produce miltiple peaks in the shear stress distribution as predicted. A similar effect would be expected on the turbulent kinetic energy. These measurements represent the most detailed set presented by Seale [6], and the good agreement with the predictions provides the confidence that the correct behaviour is being simulated. Fig. 15 compares measured and predicted vertical velocity profiles. Again we see a periodic variation in the predictions, but the measured data points are too scanty to discern any trend. Considering the measured data, Seale claims large continuity errors ( 0.19 $U_{*}$ ) along horizontal lines. Close to the horizontal wall, the imbalance was as high as $1.78 U_{*}$, which is considerably larger than the maximum secondary velocity measured. The presented data certainly do not provide an accurate representation of the flow, and have been included here only for completeness. Since the lateral velocity field is well predicted, continuity would ensure that the vertical velocity field is too. Figure 16 shows the velocity vectors and the stream function contours predicted with the NR model.The eddy formation appears to be quite complex. There are at least two eddies at each wall/wall juction, and the wall/symmetry plane junctions each contain one eddy. The two large central eddies appear to be in the process of breaking up, as has been observed in channels with large aspect ratios. The predictions with the DR model are similar, but with reduced strengths.

Predicted contours of the streamwise velocity component and the turbulent kinetic energy are compared to measured data in figures 17 and 18, respectively. The distortion of the contours due to the secondary motion is well predicted, but the quantitative agreement is only fair. The predicted components of the turbulent intensities along the vertical centreline are compared to correlations of the measured data in fig. 19. The agreement is fairly good, considering that there could be uncertainties of about $\pm 29 \%$ in the correlation coefficients [6].

It is pertinent to mention that the wall-function method used here precludes adequate resolution of the secondary velocity profiles very close to the wall. However, there is as yet no consistent Reynolds stress model for this near-wall region. Mansour et al [20], have discussed the inadequacy of the standard Launder, Reece and Rodi [14] model in this region. Rodi [21] presents a review of current attempts to bridge this gap. Preliminary calculations by the present author for a developed square duct flow, using such a near-wall model, shows that the computed mean flow and turbulence quantities are essentially the same as those obtained with the present model in the region more than $1 \%$ of the duct width, away from the wall. However, in consonance with other attempts [21], the predicted components of the Reynolds stresses do not agree with the limiting two-dimensional behaviour of turbulence in the region very close to the wall. It appears that major changes to the pressure-strain modelling would be required to overcome this problem.

\section{CONCLUDING REMARKS}

Calculation of turbulent flow in complex geometries have been presented. The performance of three turbulence models are compared to measured data for three different channel configurations. 
The standard $k-\epsilon$ turbulence model does not predict any secondary motion of Prandtl's second kind, and thus fails to predict important flow features. Although the NR model can be seen as an approximation of the DR model, it procures better quantitative agreement with measured secondary flow data. It thus enables the correct degree of redistribution of the primary velocity and the turbulence fields that takes place in complex channels to be predicted. Computations with the DR model also show the correct trends, but the magnitudes of the secondary flow is mostly underpredicted. Previous analysis had shown that differences in the treatment of the secondary shear stress are responsible for the different strengths of secondary motion predicted. Unfortunately, this stress component is the most difficult to measure, and it has received the least attention in turbulence model studies. More attention will have to be given to this component if there is to be significant progress in turbulence modelling of flow in channels with complex configuration. Coupled with this is a need for better near-wall modelling. The widely used wall-function approach now appears inadequate, for accurate simulation of flow in such complex geometries. Work is progressing on such a model. It must ensure that the various components of the Reynolds stresses exhibit the correct limiting behaviour as the wall is approached. Since detailed experimental data is limited, we have to make use of data generated by direct simulation of channel flows, such as those by Kim et al [22], and analysed by Mansour et al [20] in the light of popular Reynolds stress modelling concepts.

\section{REFERENCES}

1. Nikuradse, J.: Untersuchungen Uber Turbulente Stromungen in Nicht-Kreisformigen Rohren. Ing. Arch, vol. 1, 1930, pp. 306-332.

2. Hoagland, L.C.: Turbulent Flow in Straight Rectangular Ducts-Secondary Flow, Its Cause and Effect on the Primary Flow. PhD thesis, MIT, 1961.

3. Demuren, A.O.; and Rodi, W.: Calculation of Turbulence-Driven Secondary Motion in Non-Circular Ducts. J. Fluid Mech., vol. 140, Mar. 1984, pp. 189-222.

4. Rodet, E.: Etude de l'Ecoulement d'un Fluide Dans un Tunnel Prismatique de Section Trapezoidal. Publ. Sci. et Tech. du Min. de l'Air, No. 369, 1960.

5. Aly, A.A.M.; Trupp, A.C.; and Gerrard, A.D.: Measurements and Prediction of Fully Developed Turbulent Flow in an Equilateral Triangular Duct. J. Fluid Mech., vol. 85, pt. 1, Mar. 7, 1978, pp. 57-83.

6. Seale, W.J.: Measurements and Predictions of Fully Developed Turbulent Flow in a Simulated Rod Bundle. J. Fluid Mech., vol. 123, Oct. 1982, pp. 399-423.

7. Launder, B.E.; and Ying, W.M.: Prediction of Flow and Heat Transfer in Ducts of Square CrossSection. Proc. Inst. Mech. Eng., vol. 187, no. 37, 1973, pp. 455-461.

8. Hanjalic, K.; and Launder, B.E.: A Reynolds Stress Model of Turbulence and its Application to Thin Shear Flows. J. Fluid Mech., vol. 52, pt. 4, Apr. 25, 1972, pp. 609-638. 
9. Gessner, F.B.; and Emery, A.F.: The Numerical Prediction of Developing Turbulent Flow in Rectangular Ducts. J. Fluids Eng., vol. 103, no. 3, Sept. 1981, pp. 445-455.

10. Trupp, A.C.; and Aly, A.A.M.: Predicted Secondary Flows in Triangular Array Rod Bundles. J. Fluids Eng., vol. 101, no. 3, Sept. 1979, pp. 354-363.

11. Gosman, A.D.; and Rapley, C.W.: Fully Developed Flow in Passages of Arbitrary Cross-Section. Recent Advances in Numerical Methods in Fluids, Vol. 1, C. Taylor and K. Morgan, eds., 1980, pp. 335-399.

12. Nakayama, A.; Chow, W.L.; and Sharma, D.: Calculation of Fully Developed Turbulent Flows in Ducts of Arbitrary Cross-Section. J. Fluid Mech., vol. 128, Mar. 1983, pp. 199-217.

13. Naot, D.; and Rodi, W.: Calculation of Secondary Currents in Channel Flow. ASCE J. Hydraulic Div., vol. 108, no. 8, Aug. 1982, pp. 948-968.

14. Launder, B.E.; Reece, G.J.; and Rodi, W.: Progress in the Development of a Reynolds Stress Turbulence Closure. J. Fluid Mech., vol. 68, pt. 3, Apr. 15, 1975, pp. 537-566.

15. Demuren, A.O.; and Rodi, W.: Three-Dimensional Calculation of Turbulent Duct Flow with NonUniform Inlet Conditions. Computers Fluids, vol. 15, no. 1, 1987, pp. 47-57.

16. Rhie, C.M.; and Chow, W.L.: A Numerical Study of Turbulent Flow Past an Isolated Airfoil With Trailing Edge Separation. AIAA Paper 82-0998, June 1982.

17. Patankar, S.V.; and Spalding, D. B.: A Calculation Procedure for Heat, Mass and Momentum Transfer in Three-Dimensional Parabolic Flows. Int. J. Heat Mass Transfer, vol. 15, Oct. 1972, pp. 1787-1806.

18. Peric, M.: Efficient Semi-Implicit Solving Algorithm for Nine-Diagonal Coefficient Matrix. Numer. Heat Trans., vol. 11, no. 3, 1987, pp. 251-259.

19. Demuren, A.O.: Evaluation of Coupled and Decoupled Methods for Flows in Complex Geometries. in preparation.

20. Mansour, N.N.; Kim, J.; and Moin, P.: Reynolds-Stress and Dissipation-Rate Budgets in a Turbulent Channel Flow. J. Fluid Mech., vol. 194, Sept. 1988, pp. 15-44.

21. Rodi, W.: Recent Developments in Turbulence Modelling. Proceedings, 3rd. International Symposium on Refined Flow Modelling and Turbulence Measurements, Tokyo, 1988.

22. Kim, J.; Moin, P.; and Moser, R.: Turbulence Statistics in Fully Developed Channel Flow at Low Reynolds Number. J. Fluid Mech., vol. 177, Apr. 1987, pp. 133-166. 


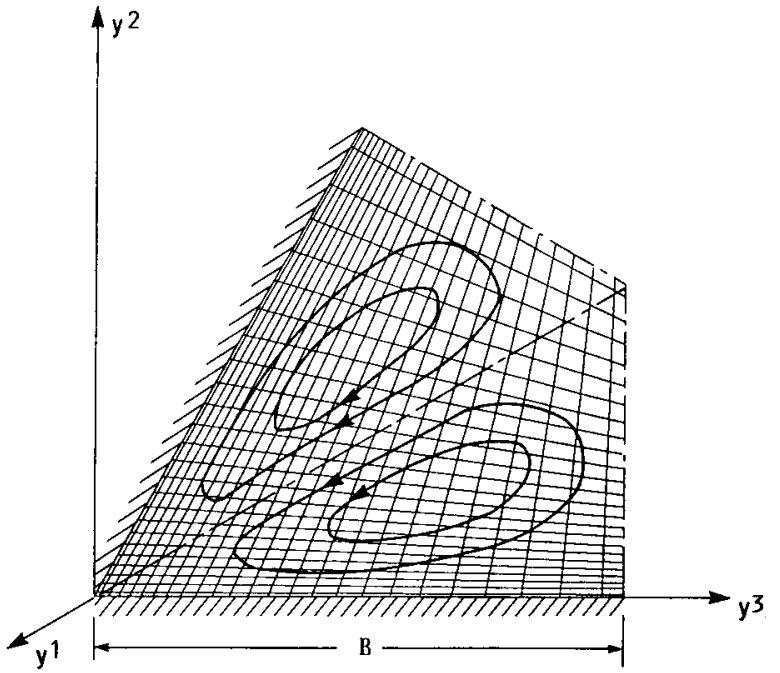

FIGURE 1. - FLOW CONFIGURATION AND GRID DISTRIBUTION FOR EQUILATERAL TRIANGULAR DUCT.

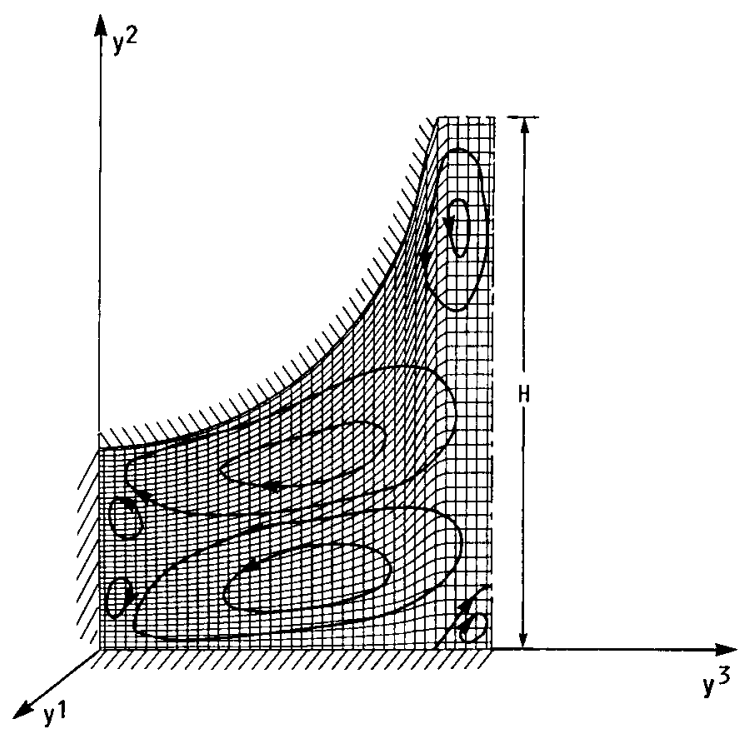

FIGURE 3. - FLOW CONFIGURATION AND GRID DISTRIBUTION FOR SIMULATED ROD-BUNDLE GEOMETRY.

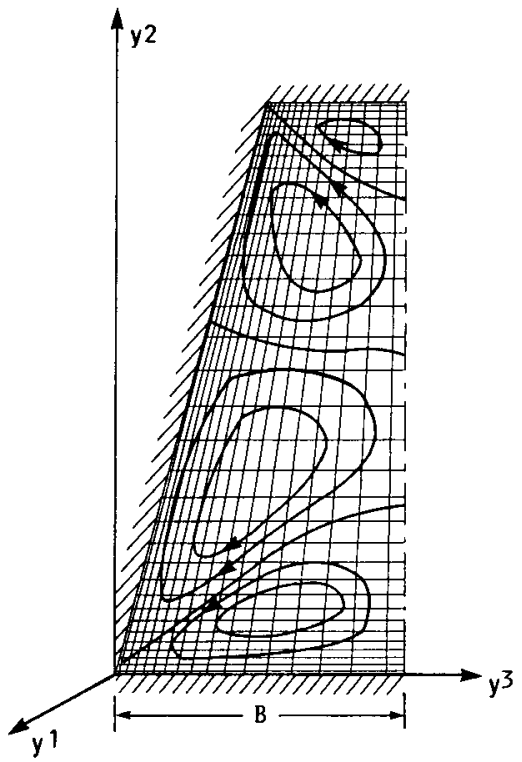

FIGURE 2. - FLOW CONFIGURATION AND GRID DISTRIBUTION FOR TRAPEZOIDAL DUCT.

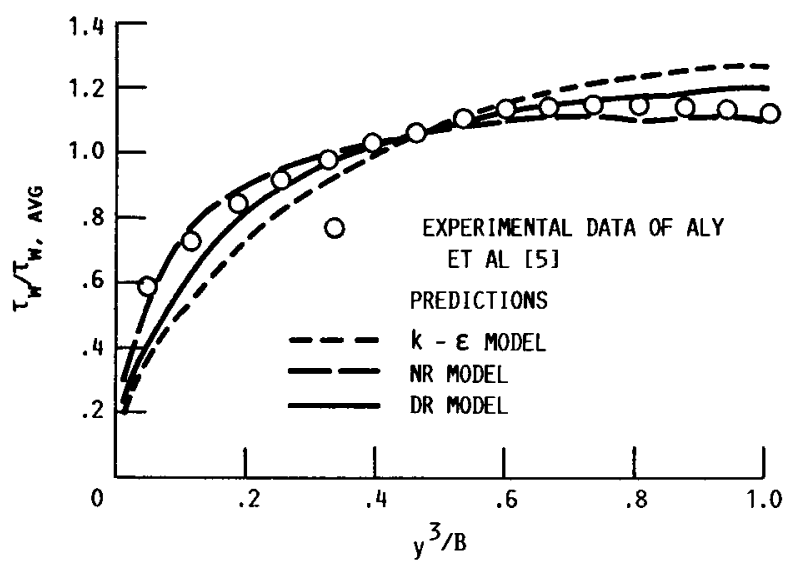

FIGURE 4. - WALL SHEAR STRESS DISTRIBUTION. 


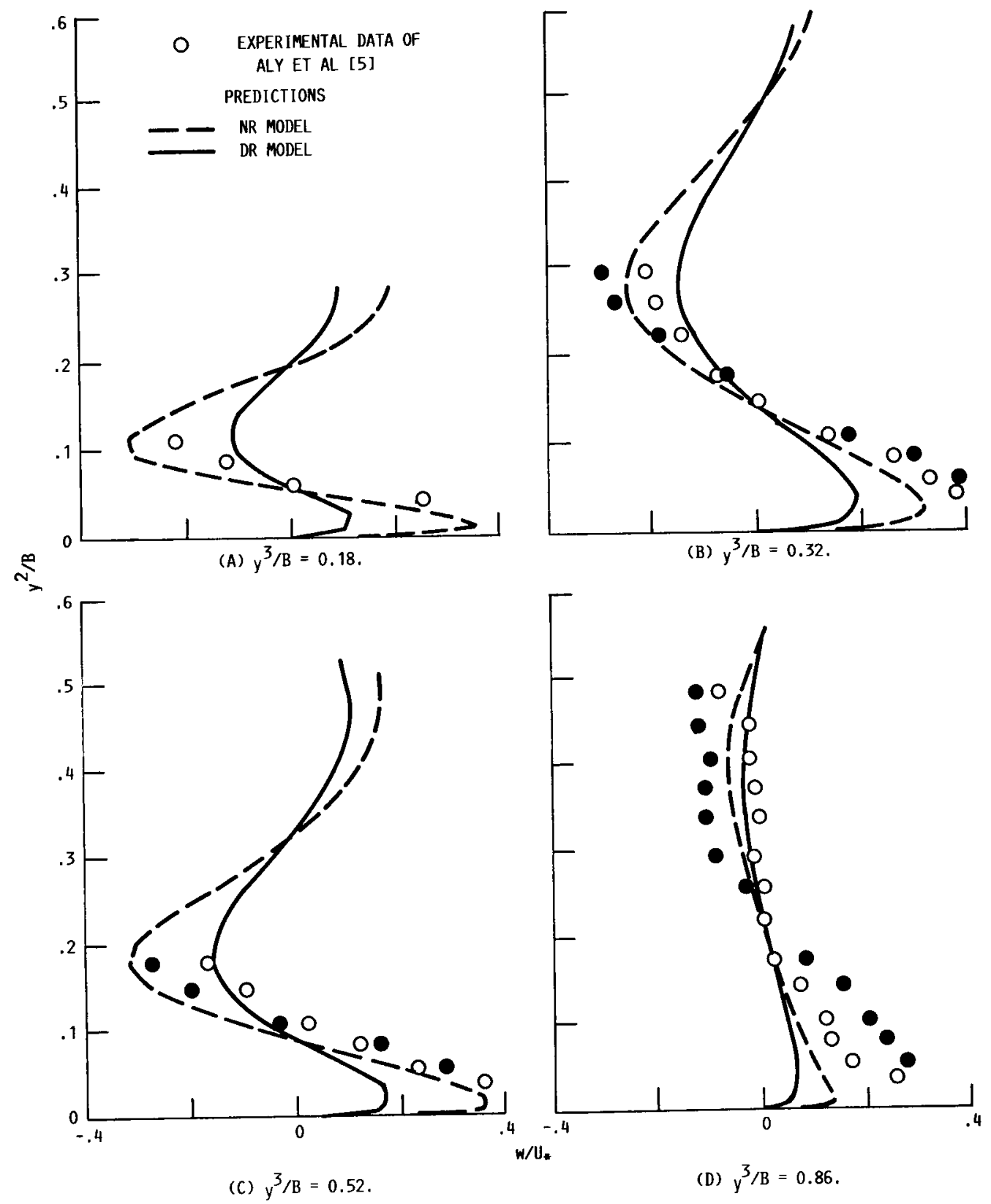

FiguRE 5. - LATERAL VELOCITY PROFILES. 


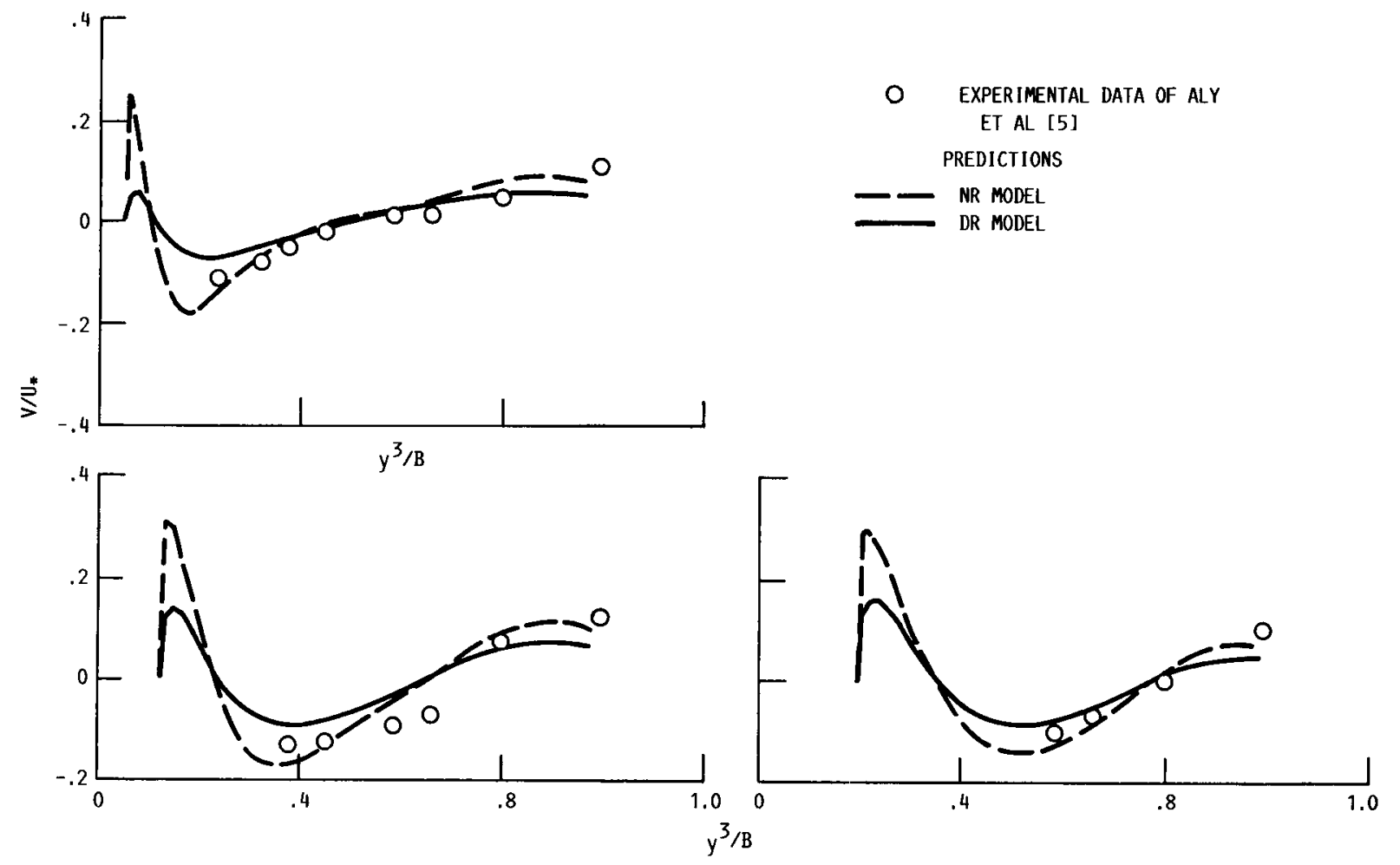

FIGURE 6. - VERTICAL VELOCITY PROFILES.

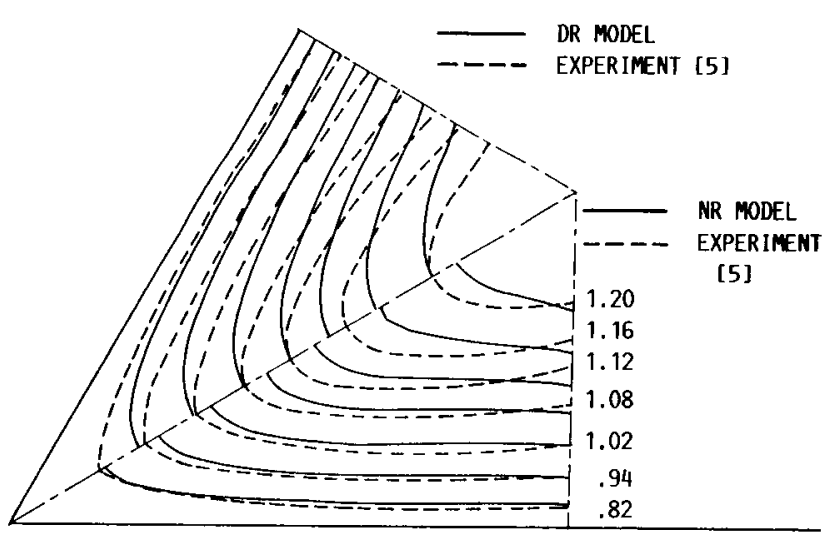

FIGURE 7. - CONTOURS OF STREAWISE VELOCITY U/U $\mathrm{u}_{\mathbf{b}}$.

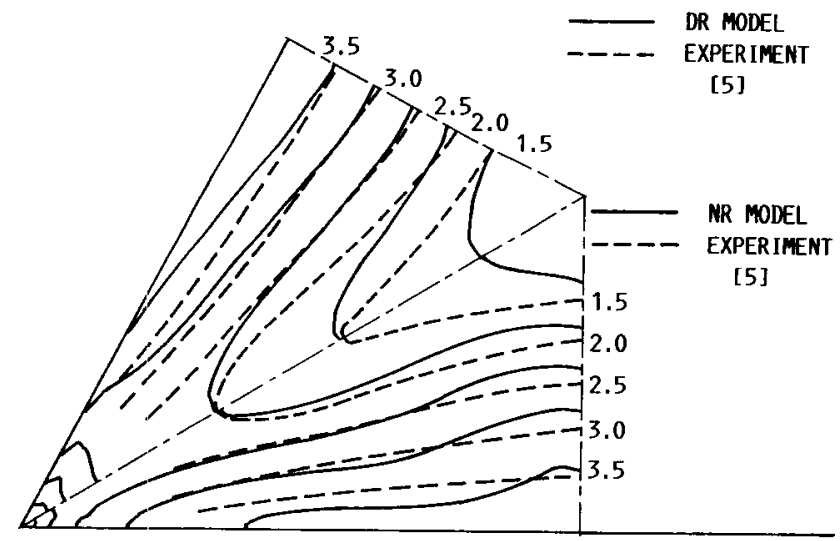

FIGURE 8. - CONTOURS OF TURBULENT KINETIC ENERGY $k /\left(\mathrm{U}_{*}\right)^{2}$. 


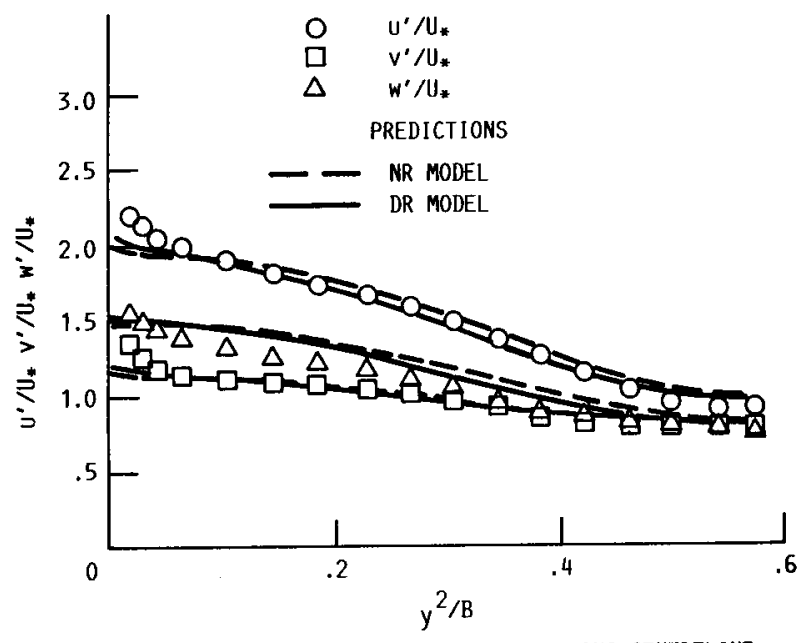

FIgURE 9. - TURBULENT INTENSITIES ALONG CENTRELINE.

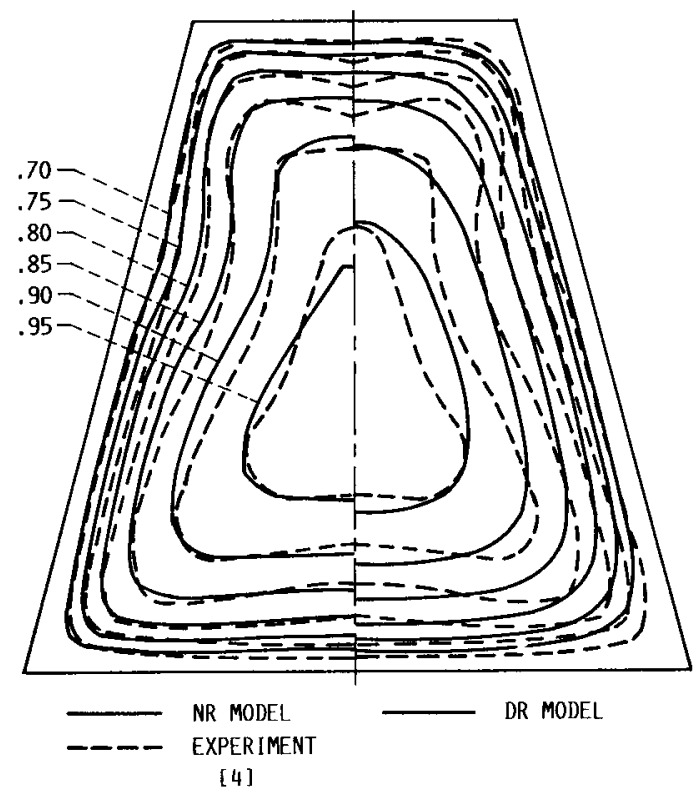

FIGURE 10. - CONTOURS OF STREAMWISE VELOCITY U/U 0 .

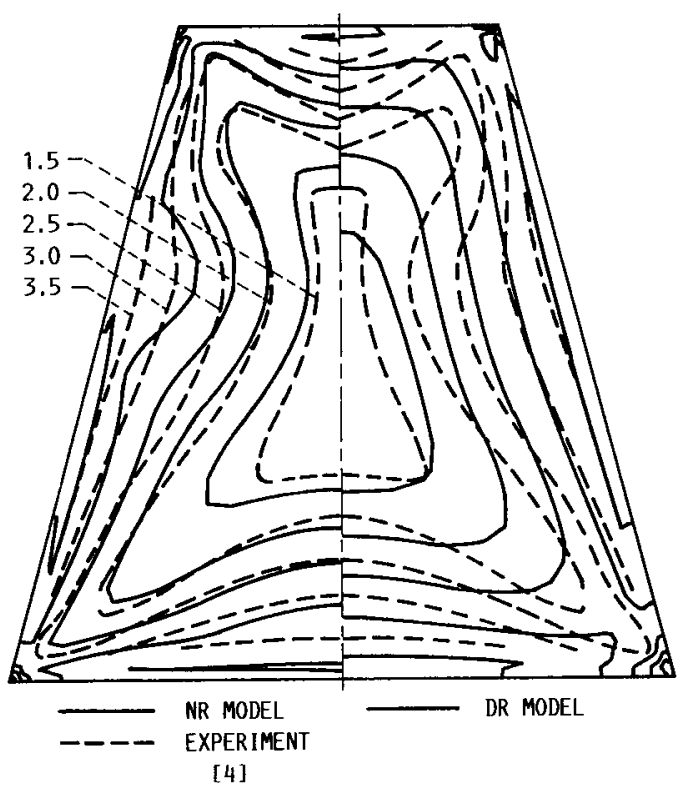

FIGURE 11. - CONTOURS OF TURBULENT KINETIC ENERGY $k /\left(U_{*}\right)^{2}$.

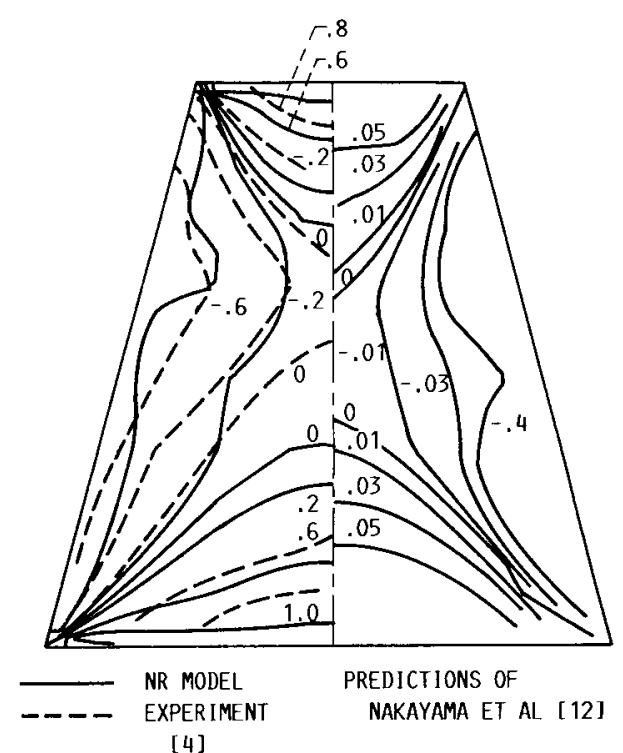

FIGURE 12. - ANISOTROPY OF THE SECONDARY NORMAL STRESSES $\left(u_{3}^{\overline{2}}-\overline{u_{2}^{2}}\right) /\left(u_{*}\right)^{2}$. 


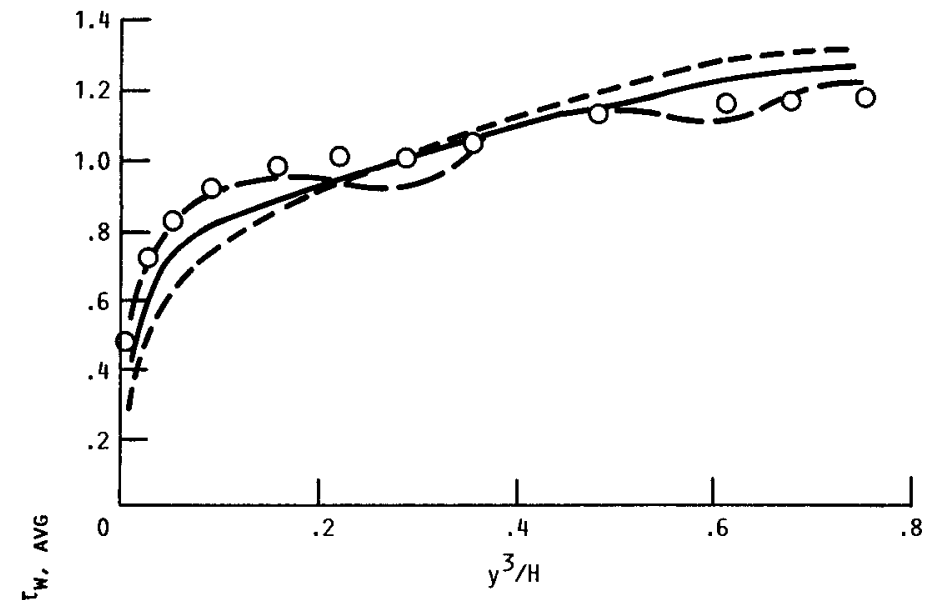

(A) HORIZONTAL WALL.

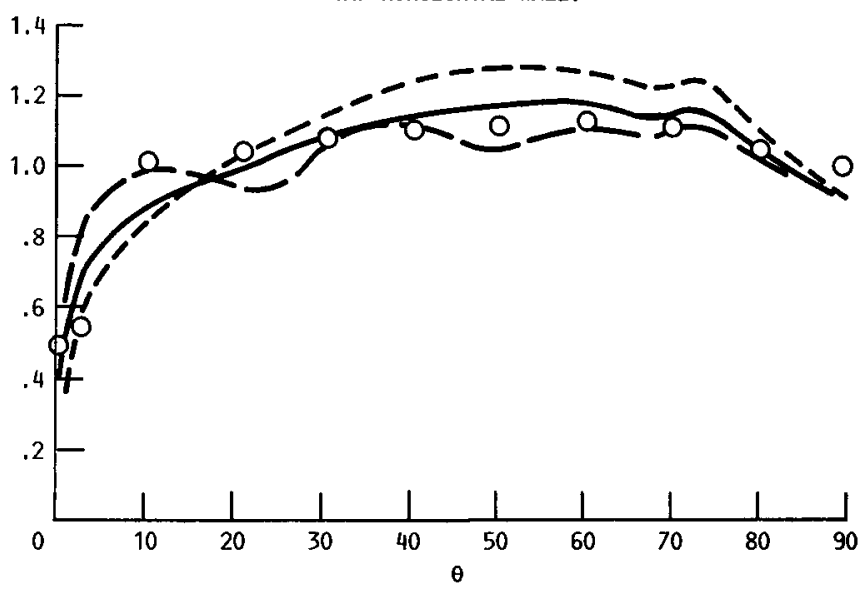

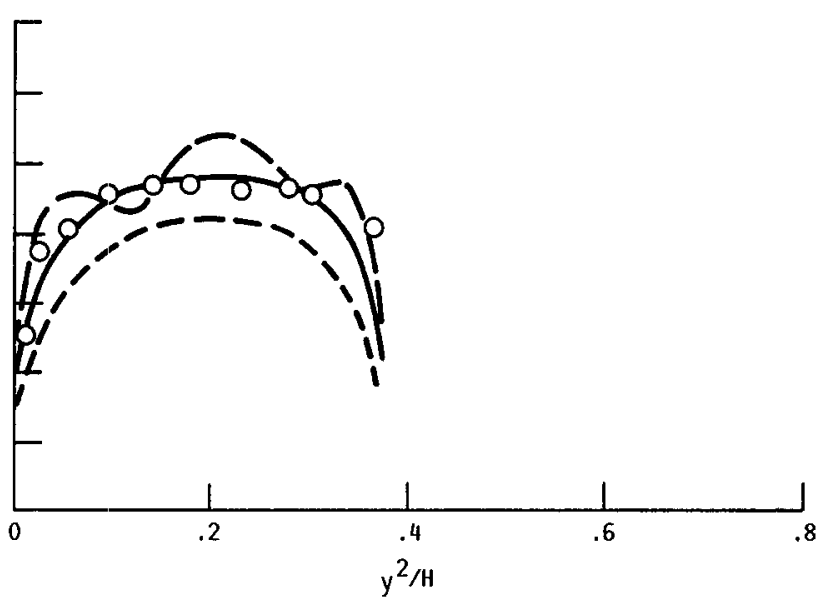

(C) VERTICAL WALL.

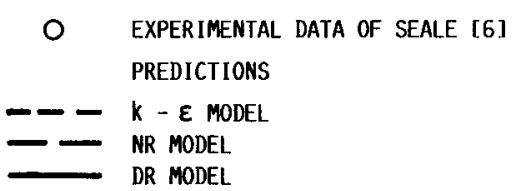
EXPERIMENTAL DATA OF SEALE [6] PREDICTIONS

NR MODEL

(B) CURVED WALL.

FIGURE 13. - WALL SHEAR STRESS DISTRIBUTION. 

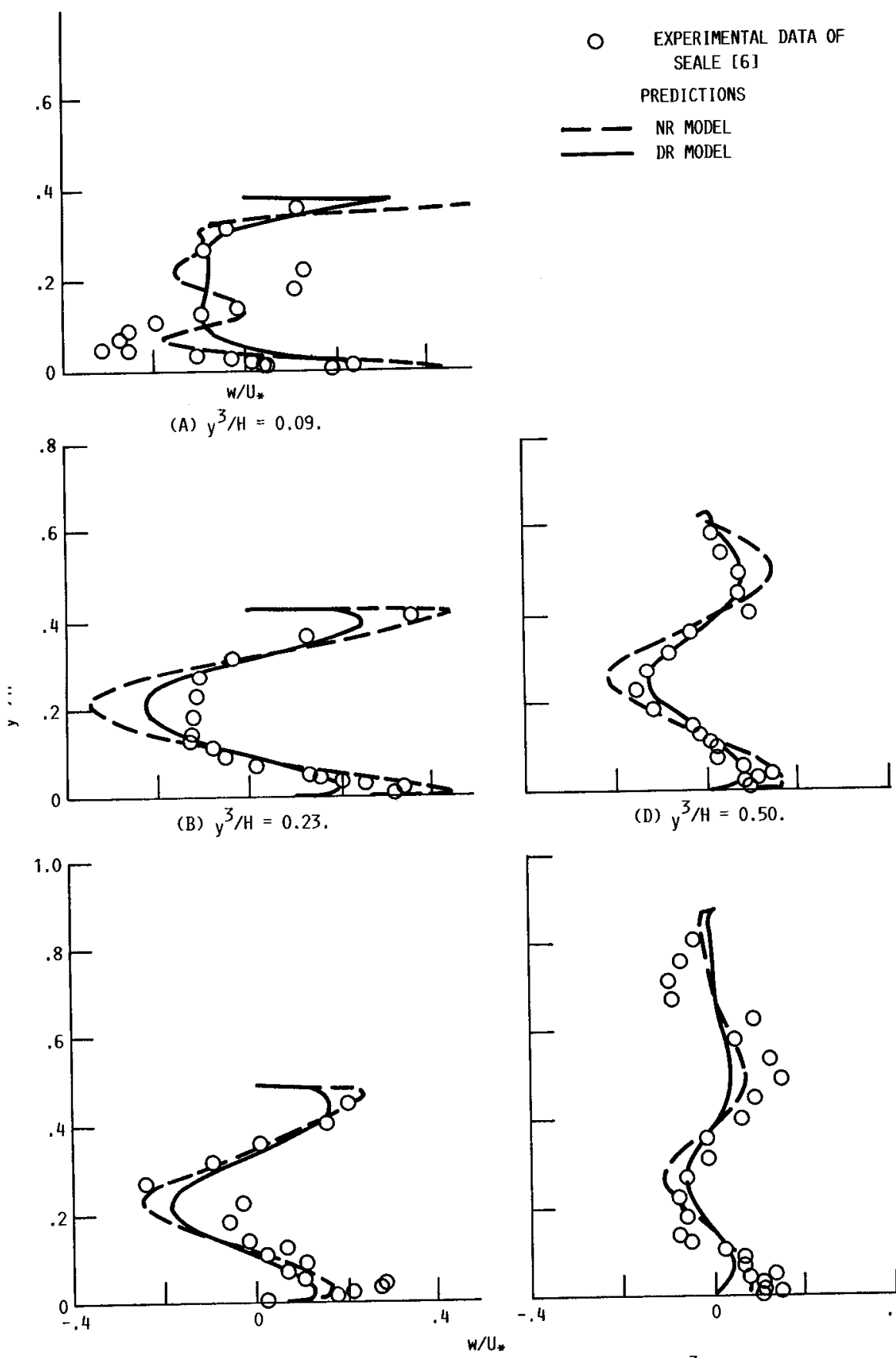

(D) $y^{3 / H}=0.50$.

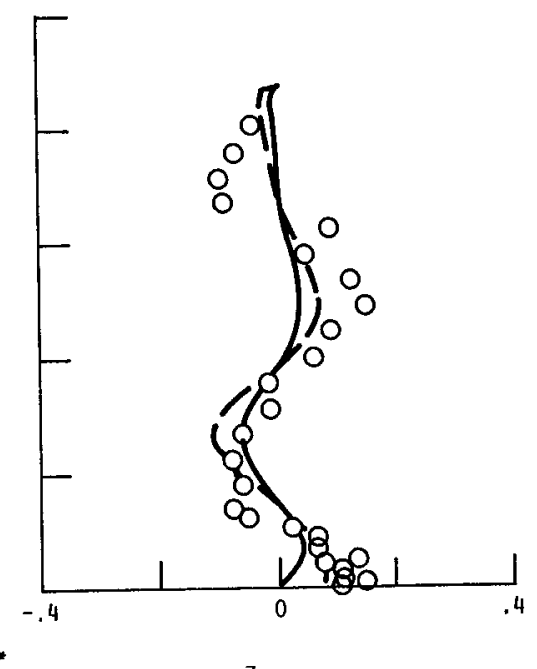

(C) $y^{3} / H=0.36$.

(E) $y^{3} / H=0.63$.

FIGURE 14. - LATERAL VELOCITY PROFILES. 

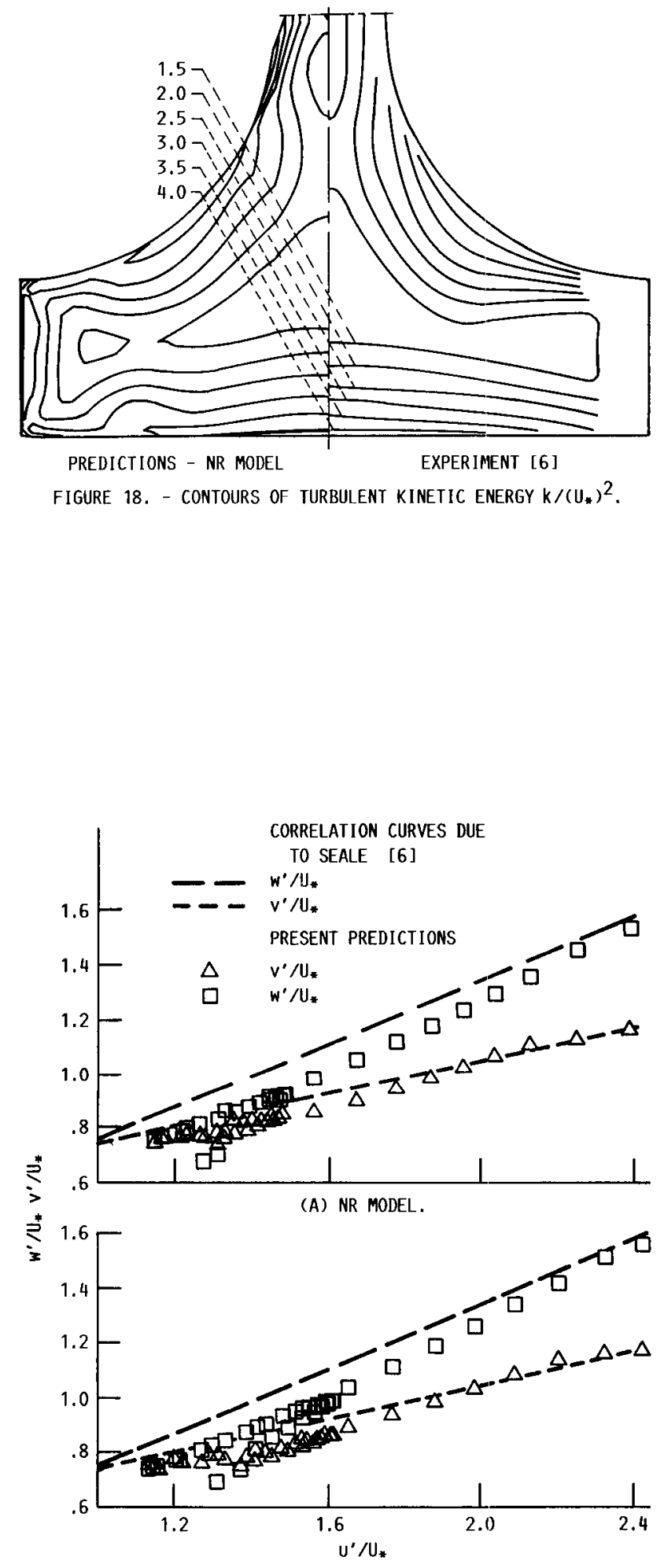

(B) DR MODEL .

FIGURE 19. - TURBULENT INTENSITIES ALONG CENTRELINE. 

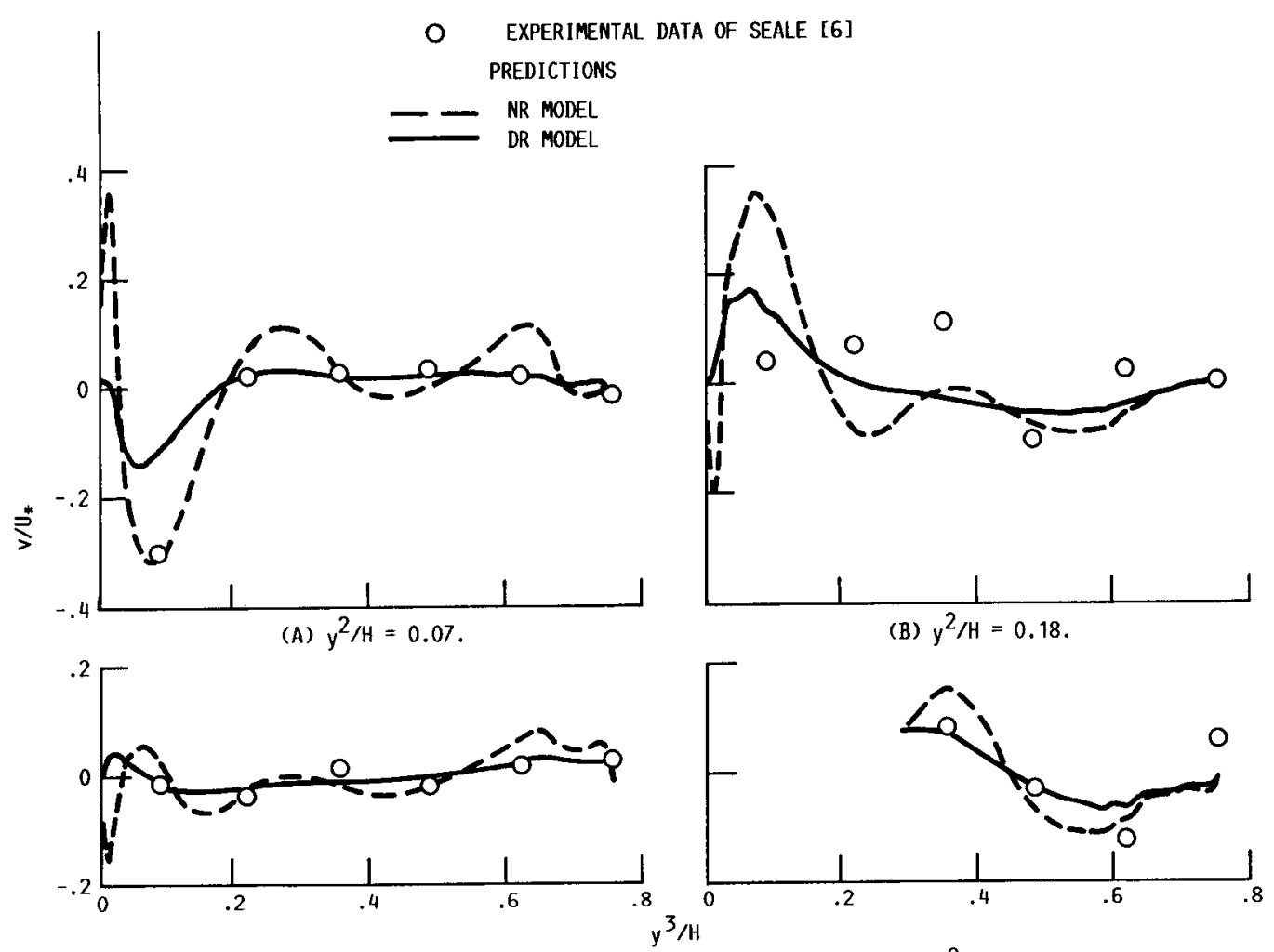

(C) $y^{2} / H=0.31$.

(D) $y^{2} / H=0.44$.

FIGURE 15. - VERTICAL VELOCITY PROFILES.
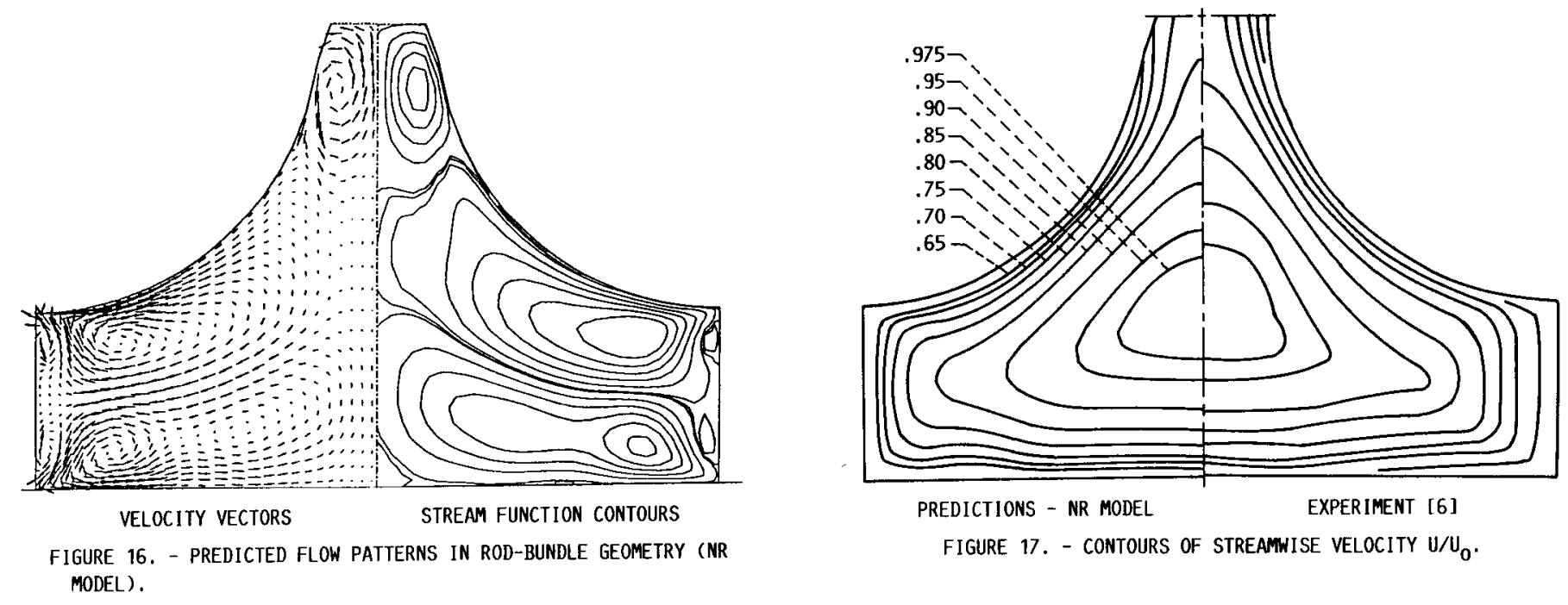


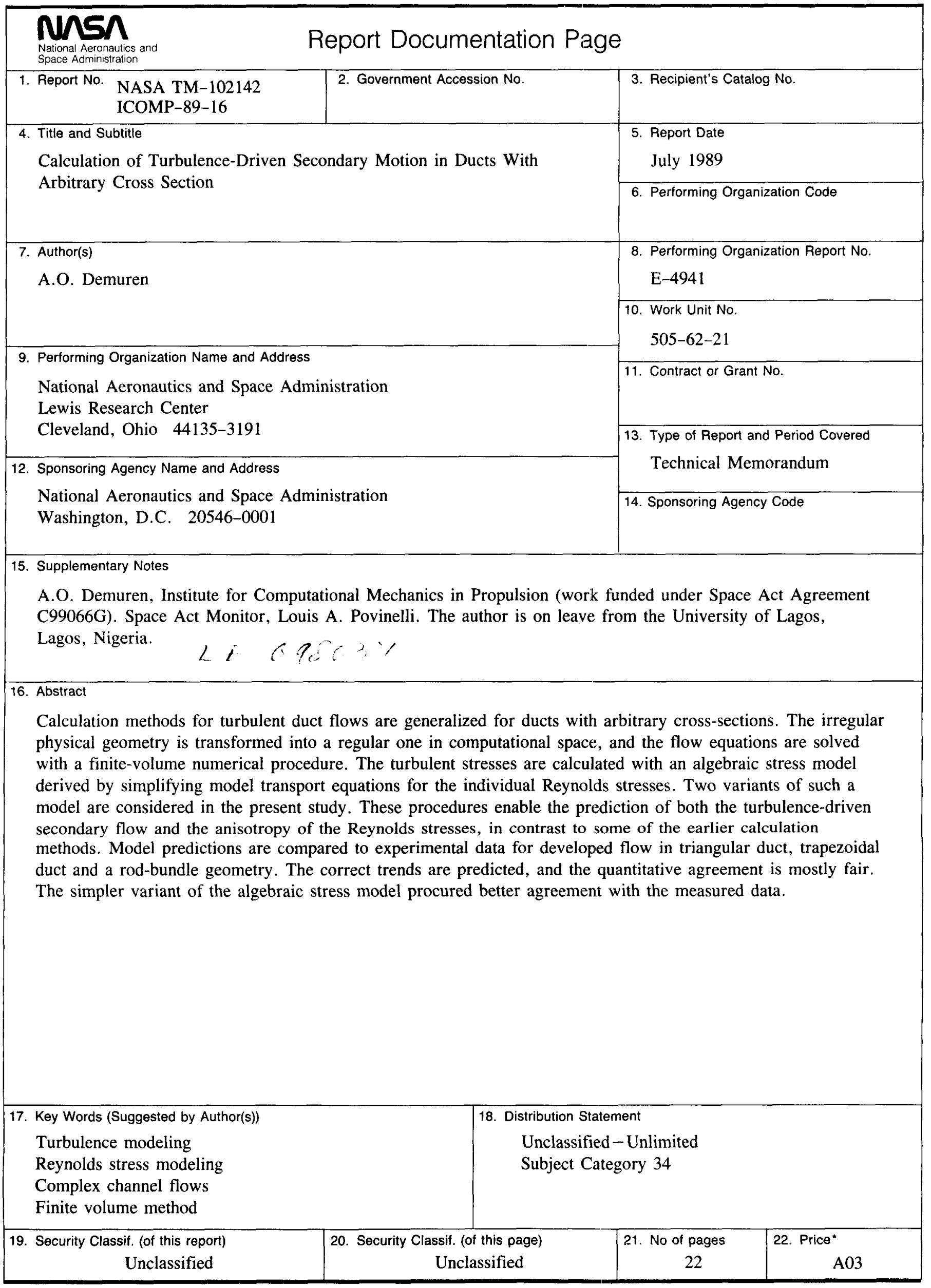

